\title{
Nature of the Vibrational-Loss EELS Peaks Measured from Ionic Specimens
}

\author{
R.F. Egerton ${ }^{1 *}$, Katia March ${ }^{2}$, Kartik Venkatraman ${ }^{3}$ and Peter A. Crozier ${ }^{3}$ \\ 1. Physics Department, University of Alberta, Edmonton, Canada T6G 2E1. \\ 2. LeRoy Eyring Center for Solid State Science, Arizona State University, Tempe, Arizona, USA. \\ 3. School for Engineering of Matter, Transport \& Energy, Arizona State University, Tempe, Arizona. \\ * Corresponding author: regerton@ualberta.ca
}

The vibrational-loss (phonon) features measured by monochromated electron energy-loss spectroscopy of ionic or partially ionic materials are dominated by dipole scattering and characterized by a narrow Lorentzian angular distribution of scattered intensity. The same features are therefore visible in infraredabsorption spectra and are fully characterized in terms of a wavelength-dependent complex permittivity.

For the case of a parallel-slab specimen, Kröger [1] provided analytical expressions for the energy-loss probability, which are easily evaluated given dielectric data in the UV, visible or IR regions [2]. Results for $\mathrm{MgO}$ and $\mathrm{SiO}_{2}$ are given in Fig. 1, for specimen thicknesses $t$ between $100 \mathrm{~nm}$ and $2500 \mathrm{~nm}$. Peaks in the energy-differential probability $\mathrm{d} P / \mathrm{d} E$ corresponding to a bulk excitation (B) scale in rough proportion to $t$, but more rapidly for thin specimens due to the begrenzungs effect [3]. Surface-mode excitations (S) show a sub-linear $t$-dependence, arising from strong coupling between the two surfaces. Plots of $\mathrm{d}^{2} P / \mathrm{d} E \mathrm{~d} t$ display an opposite behavior. Surface and bulk modes can also be distinguished on the basis of their different angular dependence of scattering, and from their peak energies, which increase with specimen thickness in the case of surface modes.

We have used a Nion HERMES monochromated STEM to measure the vibrational-peak intensities in several materials, at an incident energy of $60 \mathrm{keV}$ and with an energy resolution of $8-16 \mathrm{meV}$ (Fig. 2). Local thickness $t$ was estimated by recording the low-loss region and using the log-ratio method and the Kramers-Kronig sum rule [3]. The graph in Fig. 2 shows the probability $P_{\mathrm{LO}}=I_{\mathrm{LO}} / I_{0}$ for a hexagonal-BN specimen, $I_{0}$ being the integrated zero-loss intensity and $I_{\mathrm{LO}}$ the combined intensity of surface and bulk longitudinal-optical peaks (above background) in the energy range $0.15-0.20 \mathrm{eV}$. Elastic scattering was excluded by a $10 \mathrm{mrad}$ spectrometer-entrance aperture.

As a sum of bulk and surface peaks, $P_{\mathrm{LO}}$ is seen consist of two components. One of them is proportional to thickness and arises from the first "bulk" term in the Kröger formula. The second component derives from three surface-dependent terms and is apparently independent of thickness in the close-coupling situation $\left(t<<\lambda_{\mathrm{LO}}\right)$ that exists for thin TEM specimens. Because of the begrenzungs effect, this simple thickness scaling is not expected if intensities of the individual bulk and surface peaks are measured.

\section{References:}

[1] E. Kröger, Z. Phys. 216 (1968) 115; R. Erni and N.D. Browning, Ultramicroscopy 108 (2008) 84.

[2] E.D. Palik (ed.), Handbook of Optical Constants of Solids, vol. 1 (Academic Press, 1985).

[3] R.F. Egerton, Electron Energy-Loss Spectroscopy in the Electron Microscope (Springer, 2011).

[4] The authors acknowledge funding from the Natural Sciences and Engineering Research Council of Canada. A grant from the National Science Foundation (CHE-1508667) and use of the Nion STEM in the Eyring Materials Center at Arizona State University are also gratefully acknowledged. 

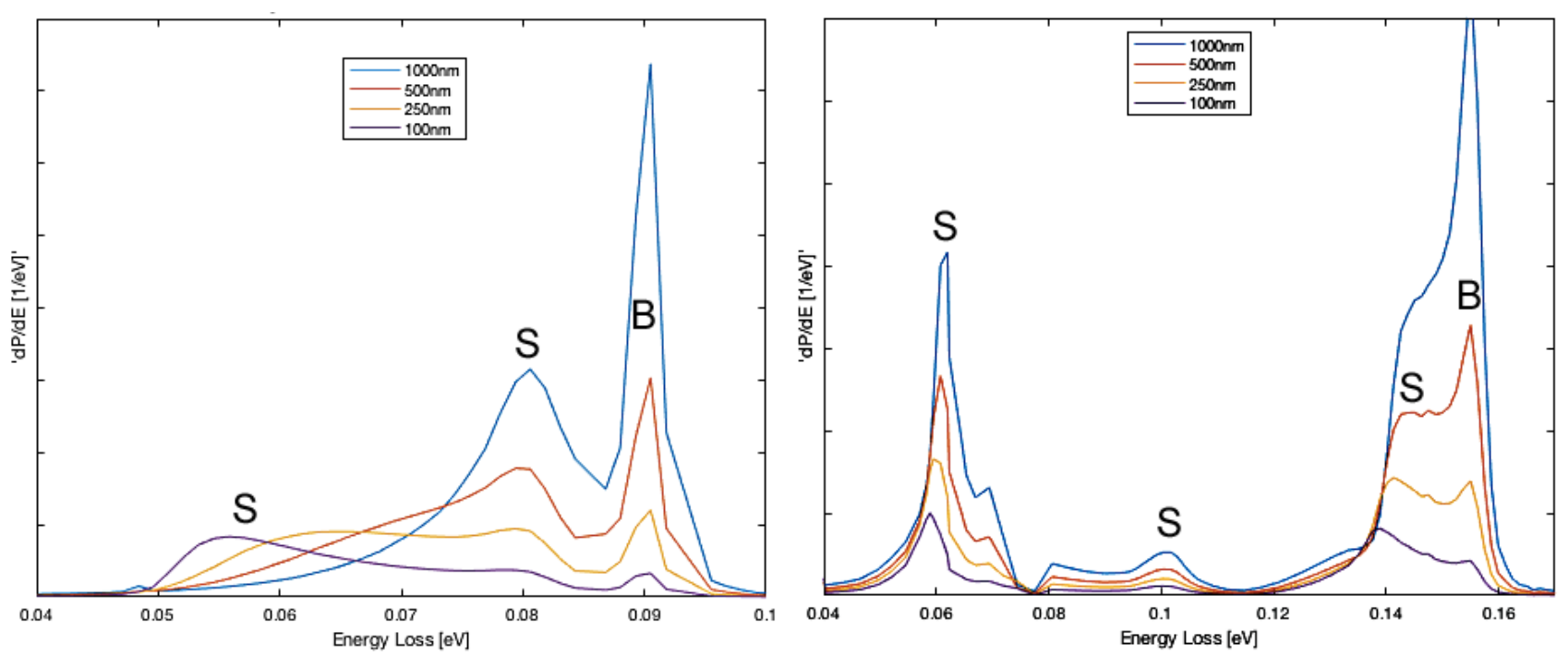

Figure 1. Predicted vibEELS spectra of $\mathrm{MgO}$ (on left) and $\mathrm{SiO}_{2}$ (on right) for film thicknesses between $100 \mathrm{~nm}$ (purple) and $1000 \mathrm{~nm}$ (blue). Peaks are labeled as surface (S) or bulk (B).
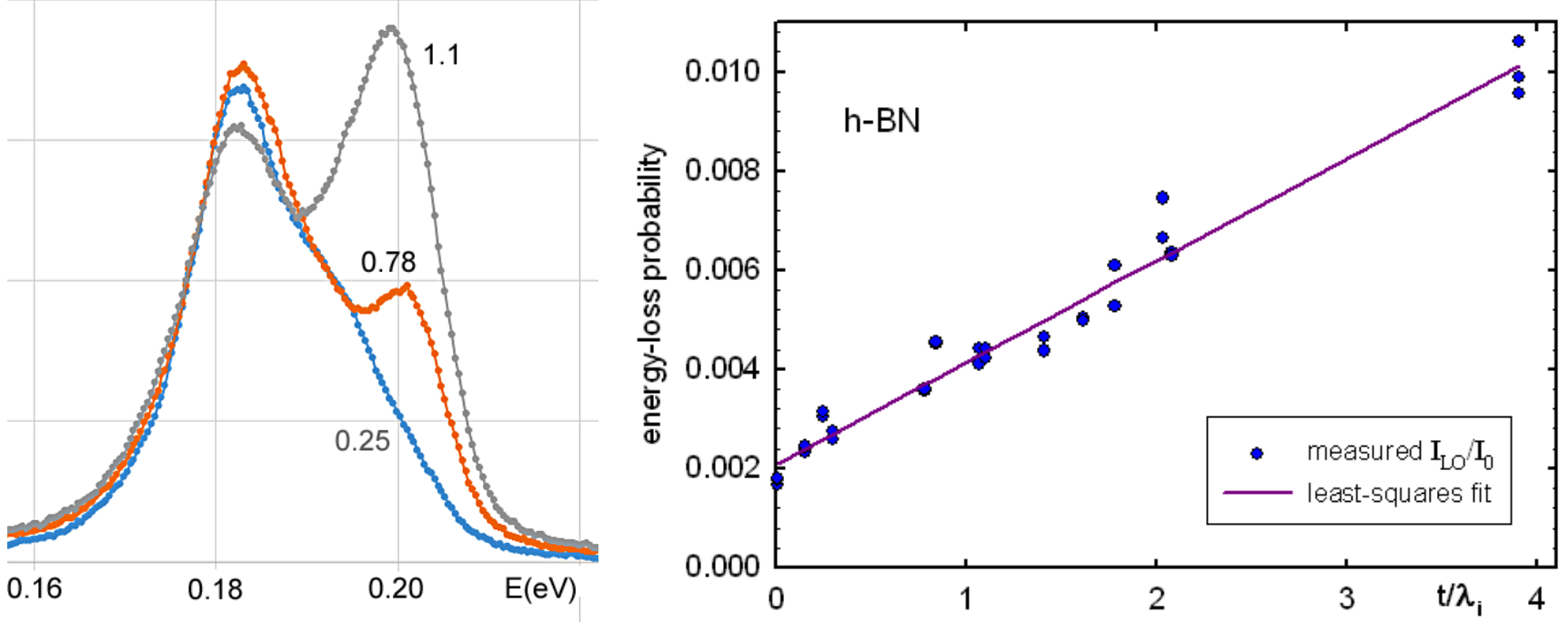

Figure 2. Left: longitudinal-optical (LO) vibrational energy-loss peaks recorded (using 60keV electrons) from regions of boron nitride of different thickness $\left(t / \lambda_{i}\right.$ between 0.25 and 1.1). The surface-mode peak occurs just above $0.18 \mathrm{eV}$ and the bulk peak at about $0.20 \mathrm{eV}$.

Right: Probability of LO excitation in h-BN (integrated over the energy range $0.16-0.21 \mathrm{eV}$ ) plotted against relative thickness: $t / \lambda_{\mathrm{i}}$ with $\lambda_{\mathrm{i}}=74 \mathrm{~nm}$ the total-inelastic mean free path. The measured slope is equal to $\lambda_{\mathrm{LO}} / \lambda_{\mathrm{i}}=1 / 500$, giving a volume mean free path $\lambda_{\mathrm{LO}}=37 \mu \mathrm{m}$. The intercept represents a thickness-independent probability: $P_{\mathrm{s}}=0.002$. 